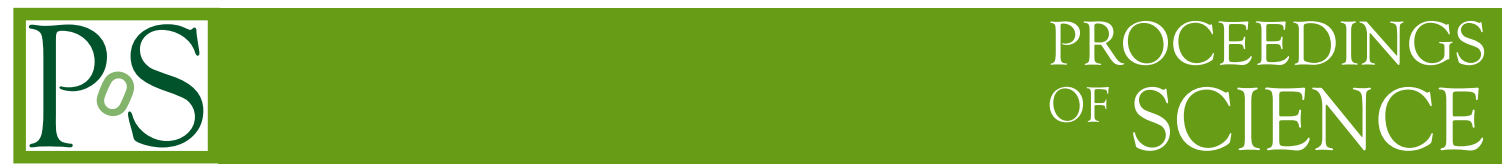

\title{
Massive and massless form factors in QCD
}

\author{
Matthias Steinhauser* \\ Institut für Theoretische Teilchenphysik, \\ Karlsruhe Institute of Technology, 76128 Karlsruhe, Germany \\ E-mail: matthias.steinhauser@kit.edu
}

We briefly review the current status of higher order QCD corrections to massless and massive form factors. Afterwards a recent calculation of massive form factors is discussed in more details.

13th International Symposium on Radiative Corrections 24-29 September, 2017

St. Gilgen, Austria

$$
{ }^{*} \text { Speaker. }
$$




\section{Form factors in QCD}

Form factors constitute a popular playground in any quantum field theory. On the one hand, they show important features as, e.g., non-trivial infrared divergences, which can be studied in detail and generalized to more complicated Greens functions. On the other hand they are sufficiently simple such that higher order quantum corrections can be computed. Form factors also enter as building blocks for virtual corrections a number of physical quantities.

For massive form factors the state-of-the-art are three-loop corrections which have been computed for the vector current in the large- $N_{c}$ limit in Ref. [1]. The complete (planar and non-planar) light-fermionic corrections are computed in Ref. [2]. Two-loop corrections for the vector current are known since more than ten years from [3] including $\mathscr{O}(\varepsilon)[4,1]$ and $\mathscr{O}\left(\varepsilon^{2}\right)$ terms [5, 6, 2]. Up to two loops the results can be expressed in terms of Harmonic polylogarithms (HPLs) [7] since only the letters 0 and \pm 1 are present. The explicit results of Refs. [8, 1] contains a further letter, which is chosen as $r_{1}=e^{i \pi / 3}$; they are expressed in terms of Goncharov polylogarithms [9]. In this context also the reduction formulae of Goncharov polylogarithm values at sixth roots of unity as derived in [10] are important.

The three-loop master integrals used in [1] have been obtained in Ref. [8]. In this reference both the exact calculation has been performed and the threshold limit has been taken to extract the hard part for $q^{2}=4 m^{2}$. These integrals enter the calculation of the matching coefficient between QCD and non-relativistic QCD which has been computed before numerically in Ref. [11] without restriction to the large- $N_{c}$ limit.

Massless form factors (below denoted by $F_{q}$ ) only depend on the virtuality of the external boson and thus, after factoring out this scale, one has to deal with a pure expansion in $\varepsilon=(4-d) / 2$. Consequently, the three-loop corrections are known since almost ten years [12, 13, 14]. Currently several groups work on the four-loop corrections. First results at four-loop order have been obtained in Refs. $[15,16]$ where all planar contributions to $F_{q}$ have been computed. This provides a complete result in the large- $N_{c}$ limit. The complete (planar and non-planar) contributions to $F_{q}$ with two closed fermion lines has been obtained in Ref. [17]. Note that the corrections with three closed quark loops have been computed in Ref. [18]. Let us also mention the works [19, 20] where the $1 / \varepsilon^{2}$ pole of the four-loop form factor within $\mathscr{N}=4$ super Yang-Mills theory has been computed using numerical methods.

In this contribution we concentrate on the discussion of the massive form factor.

\section{Massive three-loop corrections to the vector current}

The starting point in a practical calculation is the vertex function $\Gamma^{\mu}\left(q_{1}, q_{2}\right)$ which is conveniently decomposed into two scalar form factors

$$
\Gamma^{\mu}\left(q_{1}, q_{2}\right)=Q_{q}\left[F_{1}\left(q^{2}\right) \gamma^{\mu}-\frac{i}{2 m} F_{2}\left(q^{2}\right) \sigma^{\mu v} q_{v}\right]
$$

where $q=q_{1}-q_{2}$ is the outgoing momentum of the photon and $\sigma^{\mu \nu}=i\left[\gamma^{\mu}, \gamma^{\nu}\right] / 2$. $Q_{q}$ is the charge of the considered quark. Sample Feynman diagrams contributing to $\Gamma^{\mu}\left(q_{1}, q_{2}\right)$ are shown in Fig. 1. 
$F_{1}$ and $F_{2}$ are conveniently computed with the help of projectors which are applied to $\Gamma^{\mu}\left(q_{1}, q_{2}\right)$. Using the kinematics defined in Eq. (2.1) we have $(i=1,2)$

$$
F_{i}=\frac{1}{Q_{q}} \operatorname{Tr}\left\{\left(\not q_{1}+m\right)\left[a_{F_{i}} \gamma_{\mu}+b_{F_{i}} \frac{\left(q_{1, \mu}+q_{2, \mu}\right)}{2 m}\right]\left(\phi_{2}+m\right) \Gamma^{\mu}\left(q_{1}, q_{2}\right)\right\},
$$

with

$$
\begin{gathered}
a_{F_{1}}=\frac{1}{4(1-\varepsilon)\left(s-4 m^{2}\right)}, \quad b_{F_{1}}=\frac{(3-2 \varepsilon) m^{2}}{(1-\varepsilon)\left(s-4 m^{2}\right)^{2}}, \\
a_{F_{2}}=-\frac{m^{2}}{(1-\varepsilon) s\left(s-4 m^{2}\right)}, \quad b_{F_{2}}=-\frac{2 m^{2}\left(2 m^{2}+s-s \varepsilon\right)}{(1-\varepsilon) s\left(s-4 m^{2}\right)^{2}},
\end{gathered}
$$

and $s=q^{2}$. It is convenient to introduce the dimensionless variable

$$
\frac{s}{m^{2}}=-\frac{(1-x)^{2}}{x}
$$

Then the low-energy, high-energy and threshold limits correspond to $x \rightarrow 1, x \rightarrow 0$ and $x \rightarrow-1$, respectively. Note that for $x>0$ we have $s<0$ and thus the form factors do not have imaginary parts. The same is true for $x \in \mathbb{C}$ with $|x|=1$. For $0<s<4 m^{2}$ we have that $x$ is on the upper half of the unit circle.

For later convenience we write the perturbative expansion of $F_{i}(i=1,2)$ in the form

$$
F_{i}=\sum_{n \geq 0}\left(\frac{\alpha_{s}}{4 \pi}\right)^{n} F_{i}^{(n)}(x)
$$

with $F_{1}^{(0)}=1$ and $F_{2}^{(0)}=0$.

One of the first steps in the practical calculation is the definition of integral families for the massive three-loop vertices. In fact, for the large- $N_{c}$ limit ${ }^{1}$ eight families are needed which have been introduced in Ref. [8]. This information is used by q2e and exp [21, 22] which process the qgraf [23] output and generate FORM code. Let us illustrate this output for the one-loop diagram of Fig. 1 which is given by (the two- and three-loop amplitudes look very similar):

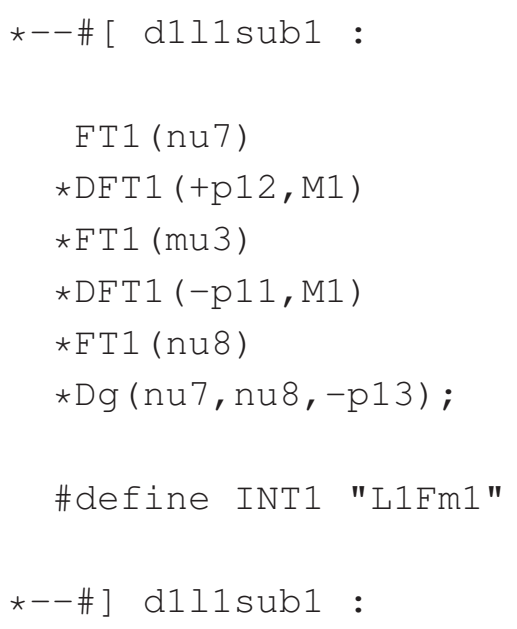

${ }^{1}$ Note that there are further planar integral families which, however, only contribute to subleading colour factors. 

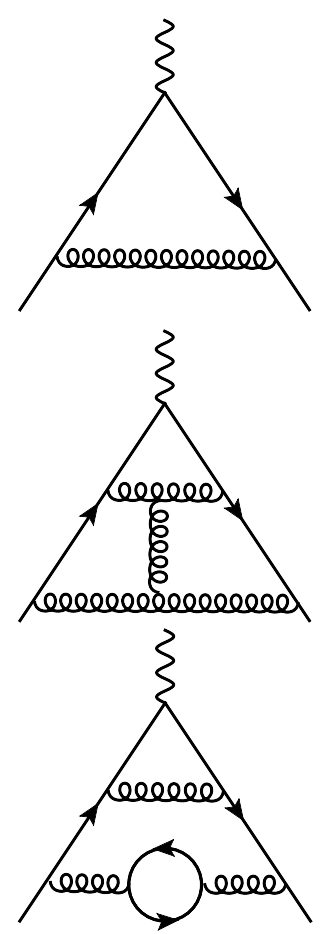
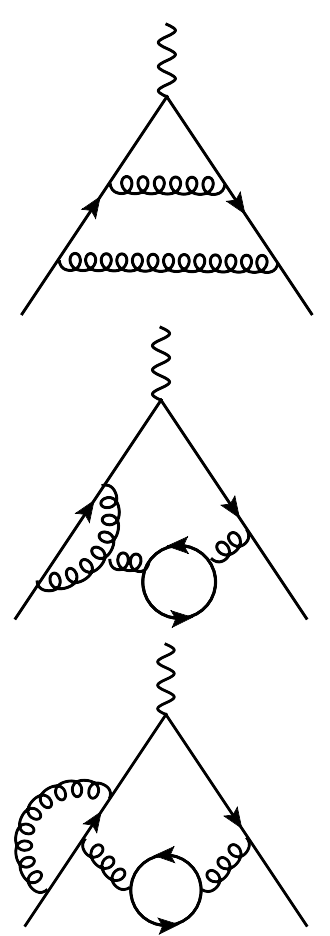
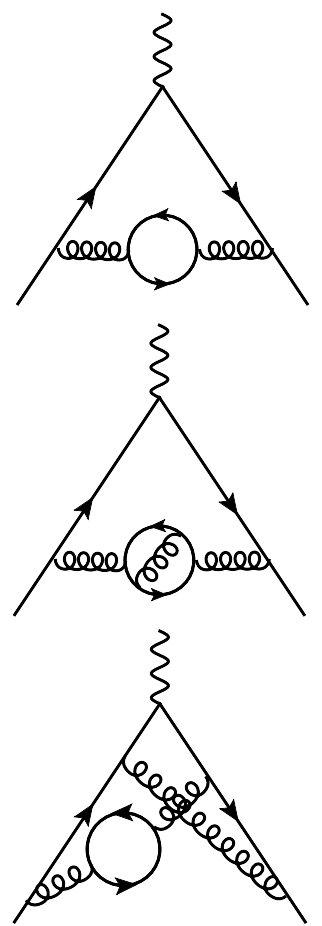
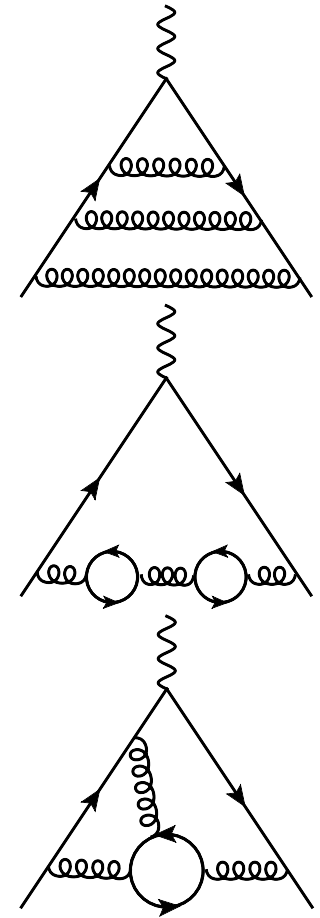

Figure 1: Sample diagrams contributing to $F_{1}$ and $F_{2}$ at one-, two- and three-loop order. Solid, curly and wavy lines represent quarks, gluons and photons, respectively.

Here, FT1 corresponds to a $\gamma$ matrix and Dg represents the gluon propagator. DFT 1 stands for the fermion propagator with mass M1 and the momentum given in the first argument. This momentum is assigned by exp according to the definition of the corresponding integral family. The preprocessor variable INT1 gets the name of the integral family as value. In the further course of the calculation a FORM file is loaded which has the name LIFm1 and which contains rules to decompose the numerator in terms of the denominator. It also identifies scalar integrals which serve as input for FIRE.

For the reduction to master integrals we use FIRE5 . 2 [24] in combination with LiteRed [25, 26]. Once the reduction for each family is complete we use the program tsort, which is part of the latest FIRE version [24] and based on ideas presented in Ref. [27], to obtain relations between primary master integrals, and to arrive at a minimal set. For the large- $N_{c}$ limit of $F_{1}$ and $F_{2}$ we need 89 master integrals. The complete fermionic corrections of Ref [2] require further 15 master integrals. Let us mention that we could perform the reduction to master integrals both for Feynman gauge and also for the linear $\xi$ term $^{2}$ (where $\xi$ is the QCD gauge parameter). $\xi$ drops out after quark mass renormalization, which is performed on-shell. In principle also the three-loop correction of the on-shell wave function renormalization constant contains $\xi$, however, not is the large- $N_{c}$ limit. The $\overline{\mathrm{MS}}$ counterterm of the strong coupling constant is $\xi$ independent.

After renormalization of the ultra-violet divergences the $n$-loop corrections to the massive form factor develops infrared poles up to order $1 / \varepsilon^{n}$ where the coefficients are determined by

\footnotetext{
${ }^{2}$ For the fermionic corrections [2] all $\xi$ terms have been considered.
} 

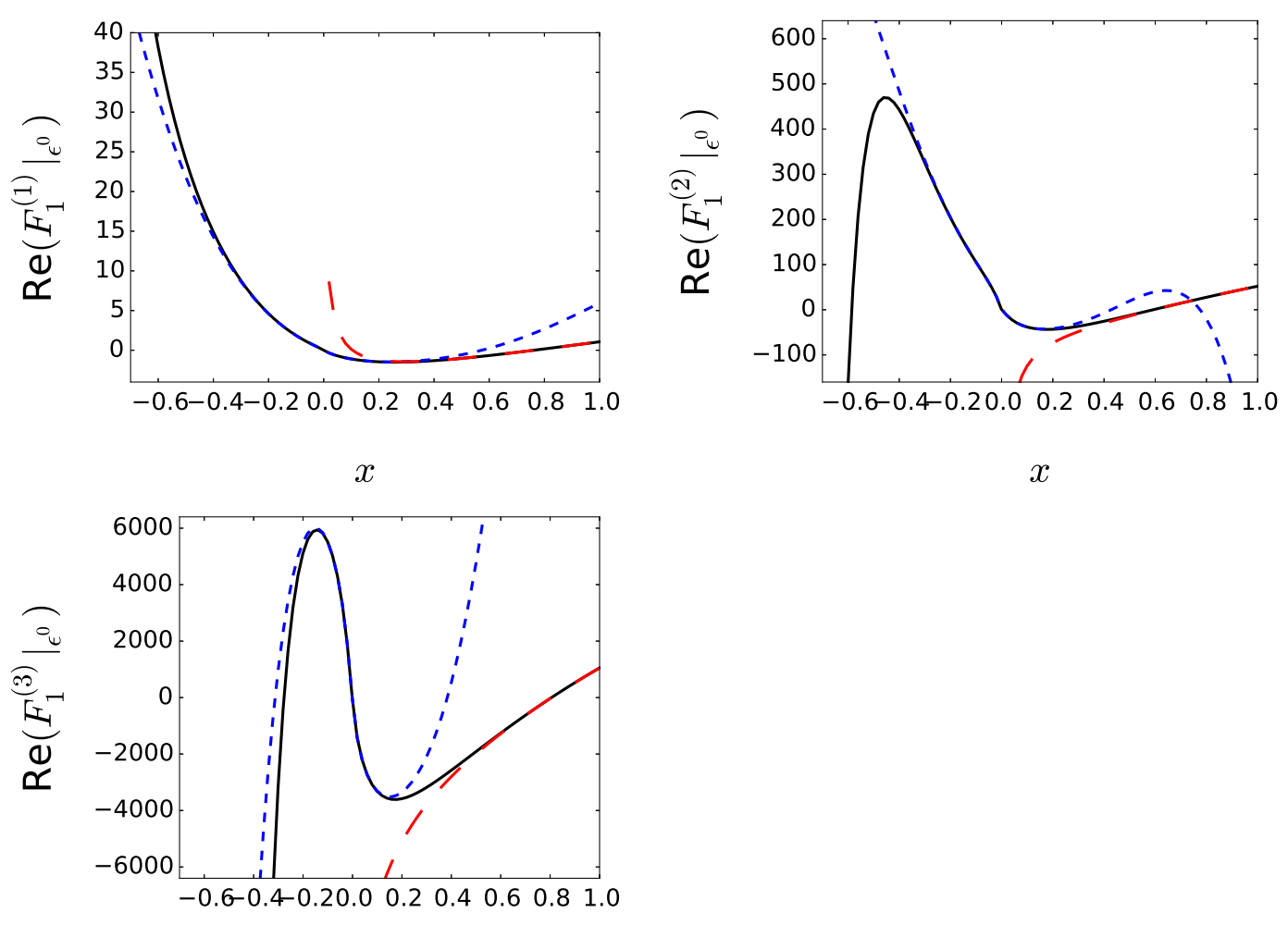

$x$

$x$

Figure 2: Real parts of $\varepsilon^{0}$ one-, two- and three-loop contribution of $F_{1}$ as a function of $x$. The leading high-energy term is subtracted so that $F_{1}$ is zero for $x=0$. The solid (black) lines show the exact result and the short-dashed (blue) lines represent the high-energy approximations including terms up to order $x^{4}$. The long-dashed (red) curves contain low-energy expansion terms up to order $(1-x)^{4}$. The number of light fermions is set to zero $\left(n_{l}=0\right)$.

the QCD beta function and the cusp anomalous dimension $\Gamma_{\text {cusp. }}$. With our explicit calculation of the form factor we provide an independent cross check of $\Gamma_{\text {cusp }}$ in the large- $N_{c}$ limit up to three loops [28, 29, 30, 31].

The analytic results for $F_{1}$ and $F_{2}$ are quite long and we refrain from printing them in this contribution. Mathematica-readable expression for the large- $N_{c}$ limit can be downloaded from https://www.ttp.kit.edu/preprints/2016/ttp16-053/ and https://www.ttp.kit.edu/preprints/2017/ttp17-023/. In these files also expansions around the three kinematic limits $x \rightarrow 0,1$ and -1 can be found. In Fig. 2 we confront the high $(x \rightarrow 0)$ and low-energy $(x \rightarrow 1)$ limits with the exact result for the $\varepsilon^{0}$ term for $F_{1}$ (the results for $F_{2}$ are very similar) at one, two and three loops. We do not show the threshold approximation which would reproduce the singularity for $x \rightarrow-1$. It is noteworthy that the approximations cover almost the whole region in $x$. A similar behaviour as for the large- $N_{c}$ limit is also observed for the complete light-fermion contribution [2]. Thus, if a fast evaluation of $F_{1}$ and $F_{2}$ is needed one could resign to the approximations without loosing noticeably precision.

To complete the massive three-loop corrections to $F_{1}$ and $F_{2}$ one has to consider also non- 
planar non-fermionic contributions. It is expected the the corresponding analytic result leaves the class of Goncharov polylogarithms and elliptic integrals appear. Still, we expect that fast and flexible numerical evaluations of the form factors along the lines of Ref. [32] are possible.

\section{Acknowledgments}

This work is supported by the Deutsche Forschungsgemeinschaft through the project "Infrared and threshold effects in QCD". The author thanks Johannes Henn, Roman Lee, Alexander Smirnov and Vladimir Smirnov for a fruitful collaboration on the subject presented in this contribution and the organizers of "RADCOR 2017" for the invitation to the conference and the pleasant atmosphere.

\section{References}

[1] J. Henn, A. V. Smirnov, V. A. Smirnov and M. Steinhauser, JHEP 1701 (2017) 074 doi:10.1007/JHEP01(2017)074 [arXiv:1611.07535 [hep-ph]].

[2] R. Lee, A. V. Smirnov, V. A. Smirnov and M. Steinhauser, in preparation.

[3] W. Bernreuther, R. Bonciani, T. Gehrmann, R. Heinesch, T. Leineweber, P. Mastrolia and E. Remiddi, Nucl. Phys. B 706 (2005) 245 doi:10.1016/j.nuclphysb.2004.10.059 [hep-ph/0406046].

[4] J. Gluza, A. Mitov, S. Moch and T. Riemann, JHEP 0907 (2009) 001 doi:10.1088/1126-6708/2009/07/001 [arXiv:0905.1137 [hep-ph]].

[5] T. Ahmed, J. M. Henn and M. Steinhauser, JHEP 1706 (2017) 125 doi:10.1007/JHEP06(2017)125 [arXiv:1704.07846 [hep-ph]].

[6] J. Ablinger, A. Behring, J. BlÃijmlein, G. Falcioni, A. De Freitas, P. Marquard, N. Rana and C. Schneider, arXiv:1712.09889 [hep-ph].

[7] E. Remiddi and J. A. M. Vermaseren, Int. J. Mod. Phys. A 15 (2000) 725 doi:10.1142/S0217751X00000367 [hep-ph/9905237].

[8] J. M. Henn, A. V. Smirnov and V. A. Smirnov, JHEP 1612 (2016) 144 doi:10.1007/JHEP12(2016)144 [arXiv:1611.06523 [hep-ph]].

[9] A. B. Goncharov, Math. Res. Lett. 5 (1998) 497 doi:10.4310/MRL.1998.v5.n4.a7 [arXiv:1105.2076 [math.AG]].

[10] J. M. Henn, A. V. Smirnov and V. A. Smirnov, arXiv:1512.08389 [hep-th]. PUBLISHED !!!

[11] P. Marquard, J. H. Piclum, D. Seidel and M. Steinhauser, Phys. Rev. D 89 (2014) no.3, 034027 doi:10.1103/PhysRevD.89.034027 [arXiv:1401.3004 [hep-ph]].

[12] P. A. Baikov, K. G. Chetyrkin, A. V. Smirnov, V. A. Smirnov and M. Steinhauser, Phys. Rev. Lett. 102 (2009) 212002 [arXiv:0902.3519 [hep-ph]].

[13] T. Gehrmann, E. W. N. Glover, T. Huber, N. Ikizlerli and C. Studerus, JHEP 1006 (2010) 094 [arXiv:1004.3653 [hep-ph]].

[14] R. N. Lee and V. A. Smirnov, JHEP 1102 (2011) 102 [arXiv:1010.1334 [hep-ph]].

[15] J. M. Henn, A. V. Smirnov, V. A. Smirnov and M. Steinhauser, JHEP 1605 (2016) 066 [arXiv:1604.03126 [hep-ph]].

[16] J. Henn, A. V. Smirnov, V. A. Smirnov, M. Steinhauser and R. N. Lee, arXiv:1612.04389 [hep-ph]. 
[17] R. N. Lee, A. V. Smirnov, V. A. Smirnov and M. Steinhauser, Phys. Rev. D 96 (2017) no.1, 014008 [arXiv:1705.06862 [hep-ph]].

[18] A. von Manteuffel and R. M. Schabinger, Phys. Rev. D 95 (2017) no.3, 034030 [arXiv:1611.00795 [hep-ph]].

[19] R. H. Boels, T. Huber and G. Yang, Phys. Rev. Lett. 119 (2017) no.20, 201601 doi:10.1103/PhysRevLett.119.201601 [arXiv:1705.03444 [hep-th]].

[20] R. H. Boels, T. Huber and G. Yang, arXiv:1712.07563 [hep-th].

[21] R. Harlander, T. Seidensticker and M. Steinhauser, Phys. Lett. B 426 (1998) 125 [hep-ph/9712228].

[22] T. Seidensticker, hep-ph/9905298.

[23] P. Nogueira, J. Comput. Phys. 105 (1993) 279; http://cfif.ist.utl.pt/ paulo/qgraf.html.

[24] A. V. Smirnov, Comput. Phys. Commun. 189 (2015) 182 doi:10.1016/j.cpc.2014.11.024 [arXiv:1408.2372 [hep-ph]].

[25] R. N. Lee, arXiv:1212.2685 [hep-ph].

[26] R. N. Lee, J. Phys. Conf. Ser. 523 (2014) 012059 doi:10.1088/1742-6596/523/1/012059 [arXiv:1310.1145 [hep-ph]].

[27] A. V. Smirnov and V. A. Smirnov, Comput. Phys. Commun. 184 (2013) 2820 doi:10.1016/j.cpc.2013.06.016 [arXiv:1302.5885 [hep-ph]].

[28] A. M. Polyakov, Nucl. Phys. B 164 (1980) 171. doi:10.1016/0550-3213(80)90507-6

[29] G. P. Korchemsky and A. V. Radyushkin, Nucl. Phys. B 283 (1987) 342. doi:10.1016/0550-3213(87)90277-X

[30] A. Grozin, J. M. Henn, G. P. Korchemsky and P. Marquard, Phys. Rev. Lett. 114 (2015) no.6, 062006 doi:10.1103/PhysRevLett.114.062006 [arXiv:1409.0023 [hep-ph]].

[31] A. Grozin, J. M. Henn, G. P. Korchemsky and P. Marquard, JHEP 1601 (2016) 140 doi:10.1007/JHEP01(2016)140 [arXiv:1510.07803 [hep-ph]].

[32] R. N. Lee, A. V. Smirnov and V. A. Smirnov, expansions near singular points,” arXiv:1709.07525 [hep-ph]. 